\title{
High-specificity bioinformatics framework for epigenomic profiling of discordant twins reveals specific and shared markers for ACPA and ACPA-positive rheumatoid arthritis
}

David Gomez-Cabrero ${ }^{1,2,3,17 \dagger}$, Malin Almgren ${ }^{1,4,8 \dagger}$, Louise K. Sjöholm,4† ${ }^{1, \text { Aase H. Hensvold }}{ }^{1,5+}$, Mikael V. Ringh ${ }^{1,4}$, Rakel Tryggvadottir ${ }^{8}$, Juha Kere ${ }^{6}$, Annika Scheynius ${ }^{18}$, Nathalie Acevedo ${ }^{7}$, Lovisa Reinius ${ }^{6}$, Margaret A. Taub ${ }^{8,9}$, Carolina Montano ${ }^{12}$, Martin J. Aryee ${ }^{13,14,15,16}$, Jason I. Feinberg ${ }^{8,11}$, Andrew P. Feinberg ${ }^{8,9,10}$, Jesper Tegnér ${ }^{1,2}$, Lars Klareskog ${ }^{1,5}$, Anca I. Catrina ${ }^{1,5}$ and Tomas J. Ekström ${ }^{1,4^{*}}$

\begin{abstract}
Background: Twin studies are powerful models to elucidate epigenetic modifications resulting from gene-environment interactions. Yet, commonly a limited number of clinical twin samples are available, leading to an underpowered situation afflicted with false positives and hampered by low sensitivity. We investigated genome-wide DNA methylation data from two small sets of monozygotic twins representing different phases during the progression of rheumatoid arthritis (RA) to find novel genes for further research.

Methods: We implemented a robust statistical methodology aimed at investigating a small number of samples to identify differential methylation utilizing the comprehensive CHARM platform with whole blood cell DNA from two sets of twin pairs discordant either for ACPA (antibodies to citrullinated protein antigens)positive RA versus ACPA-negative healthy or for ACPA-positive healthy (a pre-RA stage) versus ACPA-negative healthy. To deconvolute cell type-dependent differential methylation, we assayed the methylation patterns of sorted cells and used computational algorithms to resolve the relative contributions of different cell types and used them as covariates.

Results: To identify methylation biomarkers, five healthy twin pairs discordant for ACPAs were profiled, revealing a single differentially methylated region (DMR). Seven twin pairs discordant for ACPA-positive RA revealed six significant DMRs. After deconvolution of cell type proportions, profiling of the healthy ACPA discordant twin-set revealed 17 genome-wide significant DMRs. When methylation profiles of ACPA-positive RA twin pairs were adjusted for cell type, the analysis disclosed one significant DMR, associated with the EXOSC1 gene. Additionally, the results from our methodology suggest a temporal connection of the protocadherine beta-14 gene to ACPA-positivity with clinical RA.

(Continued on next page)
\end{abstract}

\footnotetext{
* Correspondence: Tomas.Ekstrom@ki.se

${ }^{\dagger}$ Equal contributors

${ }^{1}$ Center for Molecular Medicine at Karolinska Institutet and Karolinska University Hospital, Stockholm, Sweden

${ }^{4}$ Department of Clinical Neuroscience, Karolinska Institutet, Stockholm,

Sweden

Full list of author information is available at the end of the article
} 
(Continued from previous page)

Conclusions: Our biostatistical methodology, optimized for a low-sample twin design, revealed non-genetically linked genes associated with two distinct phases of RA. Functional evidence is still lacking but the results reinforce further study of epigenetic modifications influencing the progression of RA. Our study design and methodology may prove generally useful in twin studies.

Keywords: Rheumatoid arthritis, ACPA, DNA methylation, Epigenetics, Bioinformatics

\section{Background}

Epigenetic states define the functional genome and its communication with, and response to, the environment [1]. Importantly, epigenetic modifications have been shown to be partly associated with the genetic and environmental backgrounds in which they reside $[2,3]$. Since epigenetic mechanisms are believed to be important players in the interactions between genome and environment, it is essential to separate the genetic and epigenetic components in order to elucidate the mechanisms by which the environment may impact on the genome and phenotypes. This can be approached by studies of monozygotic twins with discordant phenotypes.

One of the basic epigenetic mechanisms is DNA methylation, which is closely associated with gene regulation both near and distant to genes. The pattern of DNA methylation of "CpG island shores", 1-2 kb downstream or upstream of $\mathrm{CpG}$ islands, has been found to associate strongly with cell type as well as disease [4]. Furthermore, distant enhancer regions may also employ methylation in gene regulatory machineries [5].

Rheumatoid arthritis (RA) is a systemic inflammatory disease affecting approximately $1 \%$ of the human population [6] with a multifactorial etiology [7, 8]. RA development has been serologically investigated using retrospective collected samples $[9,10]$, and more recently prospective collected biobank samples from individuals at increased risk for RA [11-13] have allowed identification of several distinct phases of disease development [14]. One of these initial phases is characterized by signs of deregulated immune system function with the presence of disease-specific autoantibodies-referred to as anti citrullinated peptide antibodies (ACPA)-directed to tissue antigens expressed in the joints. These antibodies can be detected already a decade prior to clinical RA symptoms [9, 15]. ACPA-positive RA disease development is therefore thought to be an accessible developmental prototype of a complex autoimmune disease. The normal maintenance of the immune system, as well as the failure to regulate it, is dependent on epigenetic factors [16]. RA is a complex autoimmune disease in which epigenetic changes have been shown to mediate previously unrecognized genetic effects [2].
ACPA-positive RA is the major form of RA, its etiology involving genetic predisposition in combination with exposure to certain environmental risk factors $[7,8,17,18]$. Twin, family, and genetic studies have shown that environmental factors make a substantial contribution, besides the genetic factors, to the development of ACPA and ACPA-positive RA [11, 19, 20]. More specifically, the risk for development of ACPA and ACPA-positive RA is associated with smoking and HLA-DRB1 gene alleles $[17,18]$. In addition, over 100 non-MHC risk alleles for ACPA-positive RA have been identified [21]. Our recent finding of associations between genotype, DNA methylation, and ACPA-positive RA within the $H L A$ cluster [2] provide genetic insight into how epigenetic regulation can mediate early stages of the disease. Yet, little is known in general of how and if environmental factors orchestrate epigenetic changes before disease onset.

In our previous work, we showed how epigenetic changes in RA can mediate previously opaque genetic differences [2]. Here we wished to understand epigenetic changes where the genome has a homogenous background by employing monozygotic (MZ) twins. In order to understand some of the mechanistic changes in RA development, we set out to examine the DNA methylation profile in two $\mathrm{MZ}$ twin sets, discordant for two different phases of disease development, by using the "comprehensive high-throughput arrays for relative methylation" (CHARM) technology, which employs 2.1 million probes [22] grouped in 43,897 genomic regions. The CHARM array also includes 4500 control probes allowing unmethylated regions to be associated, on average, with values of 0 [22]. Coverage information for the CHARM array design is depicted in Additional file 1: Figure S1.

Since the sample sizes are usually small in twin studies interrogating discordant situations, a robust methodological framework was developed to identify changes in DNA methylation with high specificity, minimizing the number of false positives (low-sensitivity). Our paired data analysis suggests that the employed MZ twin model does indeed isolate epigenetic RA determinants from genetic ones, and also may identify candidate biomarkers associated with a temporal epigenetic trajectory of disease 
development. Importantly, by estimating the proportion of the common cell types in the peripheral blood samples, we were able to distinguish phenotype-driven epigenetic changes from cell type-driven ones. Our results reveal differentially methylated loci in the twin sets that discriminate ACPA-positive healthy subjects from those with ACPA-positive RA, some of which are replicated in a previously analyzed non-twin cohort, as well as also suggesting novel associated genes.

\section{Methods}

\section{Clinical material}

DNA was obtained from five healthy MZ twin pairs discordant for ACPA and seven MZ twin pairs discordant for ACPA-positive RA (Table 1; Additional file 2: Table S1). For the replication with bisulfite pyrosequencing (see the "Statistical analysis for validation" section in the "Methods") an additional six healthy MZ twin pairs discordant for ACPA and six MZ twin pairs discordant for ACPA-positivity (Additional file 2: Table S1) were analyzed. The 24 twin pairs belong to a population-based twin cohort (Twingene) which is part of the Swedish Twin Registry $[11,23]$. Information about smoking habits, $C$ reactive protein, and occurrence of the HLA-DRB1 shared epitope (SE) are listed in Table 1. ACPA presence was tested by CCP2 ELISA assay (Immunoscan CCPlus) using the cutoff set by the manufacturer to define positive sera [11]. Each individual gave written approval for participation in the study and the ethical review board at the Karolinska Institutet approved the study.

ACPA-positive healthy: verification and discordance status ACPA-positive healthy discordant twins tested positive for ACPA (high concentration, $>75 \mathrm{AU} / \mathrm{ml}$ ) while their sibling tested negative for ACPA. None of the twins had self-reported chronic rheumatic joint disease at the time of blood collection. Also, none of these twins was identified with a discharge RA diagnosis (or other rheumatic joint disease diagnosis, e.g., polyarthritis) in the Swedish National Patient Register for a median time period of 3 years (interquartile range (IQR) 2-4) following blood collection.

\section{ACPA-positive RA: verification and discordance status}

The ACPA-positive RA discordant twins tested positive for ACPA (high concentration, > $75 \mathrm{AU} / \mathrm{ml}$ ) while their healthy siblings tested negative for ACPA. Also, these
ACPA-positive twins had self-reported RA at the time of blood sampling. The self-reported RA diagnosis was verified by both linkage to the Swedish National Patient Register and review of the medical records according to the American College of Rheumatology 1987 criteria [24]. None of the ACPA-negative siblings had selfreported chronic rheumatic joint disease at the time of blood collection. Also, none of these ACPAnegative siblings had previously been discharged with a RA diagnosis (or other rheumatic joint disease diagnosis, e.g., polyarthritis) in the Swedish National Patient Register for a median time period of 3 years (IQR 2-4) following blood collection.

\section{Sampling and DNA extraction}

The twins donated peripheral blood at outpatient clinics. Sera and tubes with whole blood were sent to Karolinska University Laboratory by overnight post and then forwarded to the KI Biobank. At the KI Biobank the DNA was extracted using the Puregene extraction kit (Gentra Systems, Minneapolis, MN, USA). After extraction the DNA was subsequently stored with a barcode at $-20{ }^{\circ} \mathrm{C}$. Quality control was done by $1 \%$ agarose gel to detect degradation. Sera was aliquoted and stored with a barcode in liquid nitrogen $\left(-180^{\circ} \mathrm{C}\right)$ at the KI Biobank.

\section{Low resolution typing HLA-DRB}

Two-digit HLA-DRB1 typing was conducted using sequence-specific primer PCR (DR low-resolution kit (2-digit); Olerup SSP, Saltsjöbaden, Sweden) and the PCR products were loaded on $2 \%$ agarose gels. To determine the specific genotype, an interpretation table was used according to the manufacturer's instructions. HLADRB1 SE alleles were defined as "01 (except "0103), "04, and ${ }^{*} 10$.

\section{DNA preparation and CHARM}

DNA (1 $\mu$ g per sample) was sheared, McrBC-digested, and gel fractionated before labeling and hybridization onto arrays covering 2.1 million CpG sites according to the protocol in [25]. CHARM is a method developed to analyze genome-wide gene-specific methylation that combines a purpose-made array design and a statistical procedure. The CHARM statistics-based algorithm first involves the identification of consecutive differential methylation sites, identifying them as candidate (differentially methylated regions (DMRs)) and second uses

Table 1 Summary information of the individuals selected for the experimental design

\begin{tabular}{|c|c|c|c|c|c|c|}
\hline Discordance type & $\begin{array}{l}\text { Number of } \\
\text { twin pairs }\end{array}$ & Females & Ever smokers & SE occurrence & $\begin{array}{l}\text { Median age (years) at } \\
\text { blood sampling (IQR) }\end{array}$ & $\begin{array}{l}\text { Median CRP at blood } \\
\text { sampling (IQR) }\end{array}$ \\
\hline ACPA-positive healthy & 5 & $80 \%$ & $30 \%$ & $60 \%$ & $63(62-74)$ & $1.7(1.2-4.2)$ \\
\hline ACPA-positive RA & 7 & $43 \%$ & $36 \%$ & $71 \%$ & $70(68-72)$ & $3.7(2.6-7.9)$ \\
\hline
\end{tabular}


a boot-strapping approach to compute a significance level for each DMR [22]. This protocol also covers CpGs in lower $\mathrm{CpG}$ density regions of the genome, in addition to CpG islands and shores, and employs a smoothing algorithm allowing correction for $\mathrm{CpG}$ density and fragment biases which may otherwise occur in methyl-enrichment or methyl-depletion DNA fractionation methods. DNA from peripheral blood cells (PBC) was analyzed to determine the locus-specific differential methylation patterns. The method is comprehensively described in [22] and briefly in Additional file 3: Methods. TS1 (Twin-set of ACPA-positive healthy discordant twins) and TS2 (Twinset of ACPA-positive RA discordant twins) were profiled separately in two batches; within each batch, all samples (e.g., healthy controls and RA individuals) were profiled together. In our analysis, and similarly to epigenome-wide association studies [26], we note the occurrence of a batch effect between TS1 and TS2, probably due to handling or processing effects; hence, we do not compare TS1 and TS2 statistically. Note that all chromosomal locations are based on the hg18 build (original CHARM design). When annotation to genes was conducted we applied liftOver to map to hg19 in order to confirm that the DMR-gene annotation was consistent between genome reference versions. We observed minor differences between DMRgene mapping in hg18 and hg19.

\section{Array pre-processing}

In the processing of CHARM arrays, several quality controls are considered: (1) the signal of background probes; (2) the standard deviation of untreated channel signals, which must be small; (3) the difference between the medians of control (CpG-free regions) and non-control probes, which must be negative; and (4) probes with a probe quality lower than 80 were discarded (see CHARM Bioconductor package for details [27]). In addition, after normalization, a quality control is applied to ensure that high correlation between samples is observed. For a more comprehensive discussion, see Additional file 3: Methods. Because DNA methylation profiling and CHARM arrays were processed in two different batches (for TS1 and TS2 associated samples, respectively), the data preprocessing and following analytical steps were performed separately for each batch.

\section{Methylation estimation and normalization}

The methp function of the CHARM Bioconductor package [27] was used to estimate methylation percentages from signal intensities. A three-step methodology was used: (1) within-sample normalization (using non-CpG probes as a reference for unmethylated DNA values); (2) between-sample normalization by subset quantile normalization; and (3) percentage methylation estimation [27]. We considered sub-quantile normalization and LOESS normalization for between- and withinsample normalization, respectively.

\section{Quality control for confounders}

Possible confounders were investigated with regard to their association with experimental design (batch, within TS1 and TS2 separately) and clinical information (age, gender, and smoking). Also, the global variability of samples was investigated using multi-dimensional scaling (MDS; Additional file 1: Figure S26.); briefly, MDS depicts in two dimensions the associations between samples. We did not identify any associations for confounders using MDS, sva, or principal component analysis in TS1 samples. However, TS2 samples grouped by age and gender (Additional file 1: Figure S26b). To investigate the possible association of age and gender with the skewness observed in TS2 (Fig. 1b), we compared the distribution of differences in methylation within female pairs and within male pairs separately; no significant difference was found using a Kolmogorov-Smirnov test $(p$ value $>0.2)$ and the top differentially methylated probes. Similar results were obtained when considering age and dividing samples into two groups: "aged more than 70 years" and "aged less than 70 years" ( $p$ value $>0.2$ ).

\section{Single probe analysis}

The same linear model used in dmrFind (CHARM Bioconductor package [27]) for DMR discovery was also used to compute $p$ values for individual probes. No single probe was found to be differentially methylated when considering a false discovery rate (FDR) $<0.20$ using the Benjamini-Hochberg FDR controlling procedure [28].

\section{DMR candidate identification}

The dmrFind algorithm is described in detail in [22] and further explanation is given in Additional file 3: Methods. When cell proportion information is considered, the percentages of neutrophils, natural killer (NK) cells $\left(\mathrm{CD} 56^{+}\right)$, and the sum of $\mathrm{CD} 4^{+}$and $\mathrm{CD}^{+} \mathrm{T}$ cells are included as covariates.

\section{Resampling-based family-wise error rate}

The CHARM algorithm provides three different statistics that are computed for each candidate DMR: (a) $a v g$, the average (across probes) percentage methylation difference; (b) max, the maximum percentage methylation difference; and (c) area.raw, the number of probes multiplied by the averaged difference of methylation, which is the default mode. By running the default mode we observed that the selection from the list of candidate DMRs was biased towards DMR with larger numbers of probes (Additional file 1: Figures S24 and S25). To correct for this bias, we estimated for each candidate DMR the 


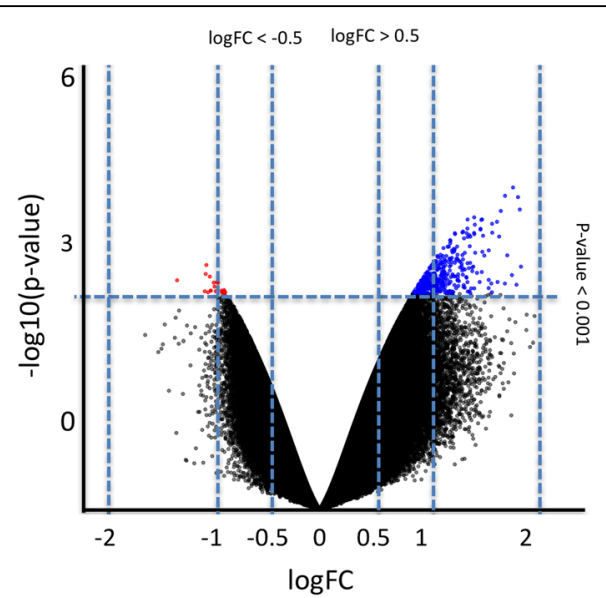

(a) TS1

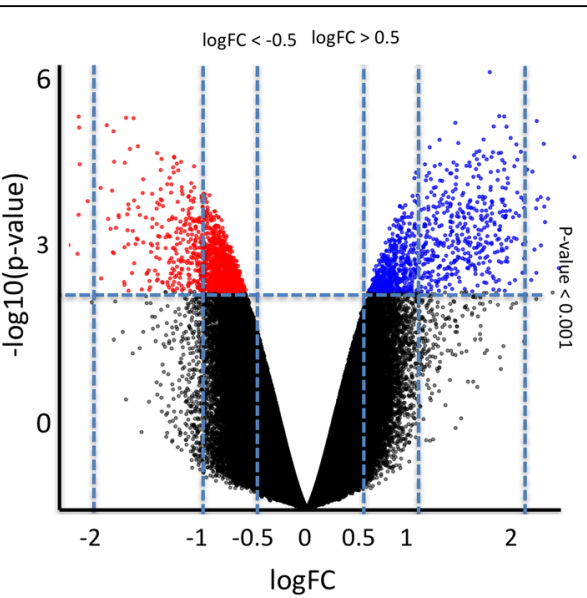

(b) TS2

Fig. 1 Differential methylation after correction for cell proportion. Log-transformed $p$ value (y-axis) versus log-transformed fold change (logFC; $x$-axis) from the associated linear model. A negative logFC denotes hypomethylation and a positive logFC hypermethylation in TS1 and TS2 comparisons; the logFC was computed using M values in a linear model and using estimated cell proportions as covariates (see "Methods"). The horizontal and vertical lines are arbitrary thresholds selected to highlight possible tendencies. a Results from ACPA-positive healthy versus ACPA-negative healthy twin siblings (TS1). b Results from ACPA-positive RA versus ACPA-negative healthy twin siblings (TS2)

family-wise error rate (FWER) similarly to CHARM FWER but considering only DMRs containing similar number of probes and selecting for each bootstrapping only those candidate DMRs (cDMRs) that are also significant in a permutation test in order to discard outlier-driven results (see the "Permuted $p$ value during bootstrapping" section in the "Methods"; Additional file 3: Methods: Bootstrapping for statistical validation). The original methodology is described in the CHARM package [27]. Briefly, FWER is computed for each DMR and for each statistic; in the case of avg it is computed as the proportion of maximum avg (across all iterations) that is greater than or equal to the $a v g$ of the DMR (similarly for max and area.raw). In both TS1 and TS2 the resampling was conducted 2000 times.

\section{Permuted $p$ value during bootstrapping}

We observed that many candidates were selected based on locus-specific outliers, i.e., a unique twin pair with extreme differences in the studied DMR but not showing differences globally and therefore not discarded as a sample. To discard these cDMRs both in the discovery run and for all bootstrapping iterations (to avoid inflation), we computed a permuted $p$ value for each DMR. For this we first computed for each sample the average (maximum) methylation over the DMR and then by randomizing the labels within each pair of twins we computed an average-associated permuted $p$ value (maximum associated permuted $p$ value). We excluded cDMRs in both the discovery run and bootstrapping iterations if the permuted $p$ value was $>0.1$ for both statistics (maximum and average). The number of permutations computed was 32 for TS1 and 128 for TS2; these numbers are limited by the number of samples.

\section{Functional analyses}

Genomic Regions Enrichment of Annotations Tool (GREAT) analysis [29] was done using the web interface provided at Bejerano's lab (http://bejerano.stanford.edu/ great/public/html/). The method defines domains for every gene and then uses this domain to map non-coding cis-regulatory regions to genes; each region may be mapped to more than one gene as domains may overlap. The purpose is to perform a functional enrichment at the gene level but overcoming the biases that may be introduced in the mapping of regions to genes; for example, genes in deserts may have larger number of regions associated with them but may not represent regulatory functions. We used GREAT version 2.0.2, with species assembly $h g 18$ and association rule Basal+extension: 5,000 bp upstream, 1,000 bp downstream, 1,000,000 bp max extension, curated regulatory domains included.

\section{Sorted cell analysis}

For cell type-specific methylation profiling, $\mathrm{CD}_{4}^{+}$and $\mathrm{CD}^{+} \mathrm{T}$ cells, $\mathrm{CD}^{+} 6^{+} \mathrm{NK}$ cells, and neutrophils were isolated from peripheral blood from five healthy male donors (mean age $\sim 38$ years) as described in Reinius et al. [30]. CHARM was performed as above.

\section{Cell proportion estimation}

To estimate cell proportion, we adapted the methodology described in [31], originally developed for the Illumina $450 \mathrm{~K}$ array. We downloaded software implementing the 
method from http://people.oregonstate.edu/ housemae/ software/. To adapt it to the CHARM array we first identified DMRs for every cell type (e.g., $\mathrm{CD} 4^{+}$samples versus rest of samples). We pooled those DMRs (sorted-cell DMRs) and generated a methylation profile for each sorted cell type by averaging the methylation of the probes within the DMRs. We then fit a regression model ("validation model" in the original notation) to select the most informative sorted cell DMRs. Next we solved a quadratic problem $(\mathrm{QP})$ in order to identify the cell proportions within our TS1 and TS2 samples. We added to the QP the condition that the sum of all the proportions was required to be 1 in order to gain stability in the results. We selected 300 sorted-cell DMRs as informative DMRs.

\section{Projection analysis}

We wanted to check if the identified DMRs in TS1 (considering cell proportion correction) were likely cDMRs in TS2 using TS2 data. To do this we used the genomic intervals (DMRs found in TS1) and computed the permuted $p$ value [32] when using data from TS2. To compute the permuted $p$ values we used two of the statistics used during DMR finding: $\max$ and average (see "Methods"); note that the area.raw statistic in this case provides similar results to the average statistic. For each statistic and for each TS1 DMR we computed a score $p$ value, where score can be $\max$ or average. Similarly, we investigated TS2 DMRs in the TS1 data.

\section{Changes in DMRs versus confounders}

Considering the low number of samples, it is not possible to include all covariates directly into the models. Hence, covariates thought to be most relevant were chosen to investigate if the methylation differences identified are associated with any relevant covariate. Four covariates were considered: age, gender, HLA epitope, and smoking. For age and gender, and considering that we are using twin samples, we investigated the association between gender and age, and the "differences in methylation" by linear modeling analysis for each DMR were computed in R. Concerning the HLA epitope and smoking covariates, linear models between methylation profiles and the variables for each DMR were computed. For each linear model the null hypothesis was the slope associated with the covariate being 0 .

\section{Statistical analysis for validation}

Significant DMRs were selected to be validated-those associated with genes PCDHB14 (DMR1), PCDHB5 (DMR_nc_06), and EXOSC1 (DMR18). Methylation analysis by bisulfite pyrosequencing was conducted in the CpG sites described in Additional file 2: Table S4. Two types of analyses were performed: technical validation and replication. First a technical validation was done with bisulfite pyrosequencing by analyzing the same individuals profiled in CHARM; the percentage of times methylation differences in twins were in agreement when comparing CHARM and bisulfite pyrosequencing was computed ("Ratio" column in Additional file 2: Table S4). Next a differential methylation analysis was performed by linear modeling using pyrosequencing data ("Technical" column in Additional file 2: Table S4) with and without deconvolution. In all cases the $p$ values were not significant, but the directions of the changes (slopes in linear models) in methylation were conserved. For cellcorrection analysis we used as covariates the cell proportion estimations computed in CHARM. Finally, using bisulfite pyrosequencing profiling, a new set of individuals were included and the analysis repeated only without deconvolution. Again, no statistically significant associations were found; however, the slope was in the opposite direction to that in the original cohort in Val5 alone.

\section{Meta-analysis}

Meta-analysis on the bisulfite pyro-sequencing data was performed by combining the (unpaired) technical verification and the replication cohort. Additional file 2: Table S4 includes two columns depicting the outcomes of combining the validation and replication samples. We used two different methodologies: (1) $p$ value based metaanalysis by the "summation of $p$ value" method [33]; and (2), effect size-based meta-analysis considering fixed effects [34] (in all effect size-based analyses residual homogeneity was not rejected, so we used a fixed effect model). Both methodologies provided very similar results, although, as expected, the effect size-based analysis was more powerful.

\section{Results}

\section{Characteristics of investigated monozygotic twins using} CHARM

We analyzed five $M Z$ twin pairs discordant for the presence of ACPA at risk for developing RA (TS1) but without known established RA disease (called "healthy" here) and seven pairs discordant for ACPA-positive RA (TS2) (Table 1). The twins with ACPA-positive RA had varying disease duration with a median of 20 years (range $0-56)$ and were all treated with disease-modifying antirheumatic drugs (DMARDs). Additional detailed information about these samples is presented in Additional file 2: Table S1.

All samples passed the CHARM array quality criteria (see "Methods"). The source of the investigated DNA was whole blood. The analysis of the CHARM arrays was done in two steps. In the first step the analysis was conducted without considering cell proportions. Results from this analysis reflect changes in DNA methylation as a result of phenotype as well as of cell type proportion 
[30, 31, 35]. This analysis provides a better framework for technical validation of the results because it is not affected by possible errors associated with cell proportion correction methodologies. In the second step, differential methylation analysis was conducted with the computationally predicted information of cell proportion changes (see "Methods"). The first step may be better suited for biomarker discovery, while the second step will provide a basis for hypotheses pertaining to the disease pathology.

\section{Analyses of cell type-driven differential methylated positions and regions}

When considering differential methylation only at the probe level, no single differentially methylated position (DMP) was identified at a FDR of $<0.20$ when comparing ACPA-positive healthy versus their respective ACPAnegative healthy twin siblings (TS1). Neither did we identify any statistically significant DMP when comparing ACPA-positive RA versus their respective ACPA-negative healthy twin sibling (TS2). These results align with the power analysis done for case-control studies in twins [36]. Volcano plots in Additional file 1: Figure S2a (TS1) and S2b (TS2) show the genome-wide differential methylation at the probe level for the two twin sets. We observed a larger number of hypermethylated $\mathrm{CpG}$ sites in the ACPA-positive healthy twin siblings in TS1 (Additional file 1: Figure S2a), while ACPA-positive RA individuals (TS2) show the opposite (Additional file 1: Figure S2b). We did not find statistical evidences of the skewness to be associated with confounders (see the "Quality control for confounders" section in the "Methods"). However, we identified age and gender as relevant covariates to investigate in the candidate DMRs.

To identify DMRs, we defined a high-specificity and low-sensitivity approach, aimed at prioritizing the identification of true positives and minimizing false positives when using the current small number of samples. We estimate the significance of a candidate DMR globally by computing the family-wise error rate (FWER) using an adapted bump-hunter-based algorithm as described by Jaffe et al. [37] (see the "Resampling-based family-wise error rate" section in the "Methods"). Two modifications were made: first, we take into consideration the number of probes of the candidate DMRs (cDMRs) and only include bootstrap-based cDMRs that are significant when computing a permuted $p$ value (by randomizing the labels within the twin pairs; see the "Permuted $p$ value during bootstrapping" section in the "Methods") [38]. Our second modification filters out CDMRs that are significant based only on locus-specific outliers, i.e., a unique twin pair with extreme differences in the studied DMR but not showing differences globally and therefore not discarded as a sample.
By employing these strict criteria, one DMR, associated with the protocadherin (PCDH) gene PCDHB14, was identified as significant in the TS1 group (Additional file 2: Table S2) after filtering for FWER $\leq 0.10$. The limited number of DMRs is expected in the heterogeneous cell population since all individuals are in fact healthy.

The analysis of TS2, a group where substantial differences in cell type composition is expected, revealed six significant DMRs (Additional file 2: Table S2) after filtering for FWER <0.10. In TS2, another gene in the $P C D H$ cluster was found to be differentially methylated (Additional file 1: Figure S3 for PCDHB5).

Bisulfite pyrosequencing was used for technical validation of a few selected loci (e.g., PCDH5 in Additional file 1: Figure S3). None of our technical validations achieved statistical significance, although all changes computed used pyrosequencing data were in the same direction as the CHARM results (we will refer to this as the change being directionally consistent; see the "Statistical analysis for validation" section in the "Methods"). For an initial technical validation, we selected cDMRs associated with (FWER $<0.20)$ COL13A1 (Additional file 1: Figure S4) and SLITRK2 (Additional file 1: Figure S5) genes because the methylation differences were large enough to be analyzed by pyrosequencing; in both cases the changes were in the same direction as those observed in the CHARM data. Additionally, we performed a technical replication by bisulfite pyrosequencing of DMRs associated with PCDHB14, PCDHB5, and EXOSC1 (Additional file 2: Table S4); we selected them based on their biological relevance and in all cases we observed the change is directionally consistent. Finally, we conducted bisulfite pyrosequencing of the same CpGs associated with $P C D H B 14, P C D H B 5$, and EXOSC1 in independent replication cohorts; in all but one case did we observe changes to be directionally consistent (Additional file 2: Table S4). Finally, we conducted a meta-analysis on the pyrosequencing data by combining the technical validation data and the replication cohort data. For this, we used two different methodologies (see the "Meta-analysis" section in the "Methods") that returned similar results and between one and three significant CpGs $(p$ value $<0.05$; Additional file 2: Table S4) associated with $P C D H B 5$ (in TS2) and PCDHB14 (in TS1).

Several of the TS2 DMRs identified were associated with regions that differentiate the methylation profiles of $\mathrm{CD}_{4}{ }^{+} \mathrm{T}$ cells and neutrophils (see "Methods" and an example in Additional file 1: Figure S6 for PCDHB5). Although important for the disease phenotype, these results point in the direction of substantial methylation changes being due to differences in cell proportion between ACPA-positive RA and healthy individuals, which is consistent with our previous report [2]. 


\section{Analysis of phenotype driven differentially methylated regions: comparison with a non-twin cohort}

In order to identify differentially methylated regions caused by changes in cell type proportion, we repeated the statistical analysis while considering the cell type proportion of each sample (cell type deconvolution/correction). To do this, we used the strategy depicted in Fig. 2. As a first step we analyzed the methylation profile by CHARM in physically sorted $\mathrm{CD}_{4}^{+} \mathrm{T}$ cells, $\mathrm{CD} 8^{+} \mathrm{T}$ cells, neutrophils, and $\mathrm{CD}^{2} 6^{+} \mathrm{NK}$ cells from five healthy individuals. Those profiles allowed us to identify DMRs characteristic of each of these cell types (see the "Sorted cell analysis" in the "Methods"). By combining those DMRs we adapted an existing and validated computational procedure $[2,31]$ to generate robust estimations of the cell proportions in each sample (see the "Cell proportion estimation" section in the "Methods"). As a second step we applied the same DMR-finder methodology used for the "non cell-corrected analysis" (see the "DMR candidate identification" section in the "Methods") but this time we included as covariates the estimated cell proportions.

When comparing cell proportions (Table 2) we did not observe statistically significant differences after a $t$-test analysis and after correction for multiple testing, but we did observe larger proportions of neutrophils in most ACPA-positive RA twins (five of seven) compared to their ACPA-negative healthy siblings (TS2), which supports our estimations observed previously [2]. By combining results from [2] and our current data (although not significant) showing the same directionality, we conclude that correction for cell proportion is necessary. We also observed a non-significant decreased population of $\mathrm{CD} 56^{+} \mathrm{NK}$ cells in the ACPA-positive healthy samples in the TS1 group.

When considering differential methylation only at the probe level after correction for cell proportion, no single DMP was identified at a FDR $<0.20$ in either TS1 or TS2. Volcano plots in Fig. 1a (TS1) and 1b (TS2) show the genome-wide differential methylation at the individual probe level for the two twin sets. We observed a larger number of hypermethylated CpG sites in both comparisons.

However, DMR analysis in the TS1 comparison after cell type correction returned 17 DMR candidates (FWER $\leq 0.10$; see "Methods"; detailed list in Table 3). Of those, 14 DMRs were found in either $\mathrm{CpG}$ shores or $\mathrm{CpG}$ islands (Table 4; Fig. 3; Additional file 1: Figure S7-S23).

From the 17 TS1 DMRs, 13 overlapped with probes present in the Illumina $450 \mathrm{~K}$ array; ten of the 13

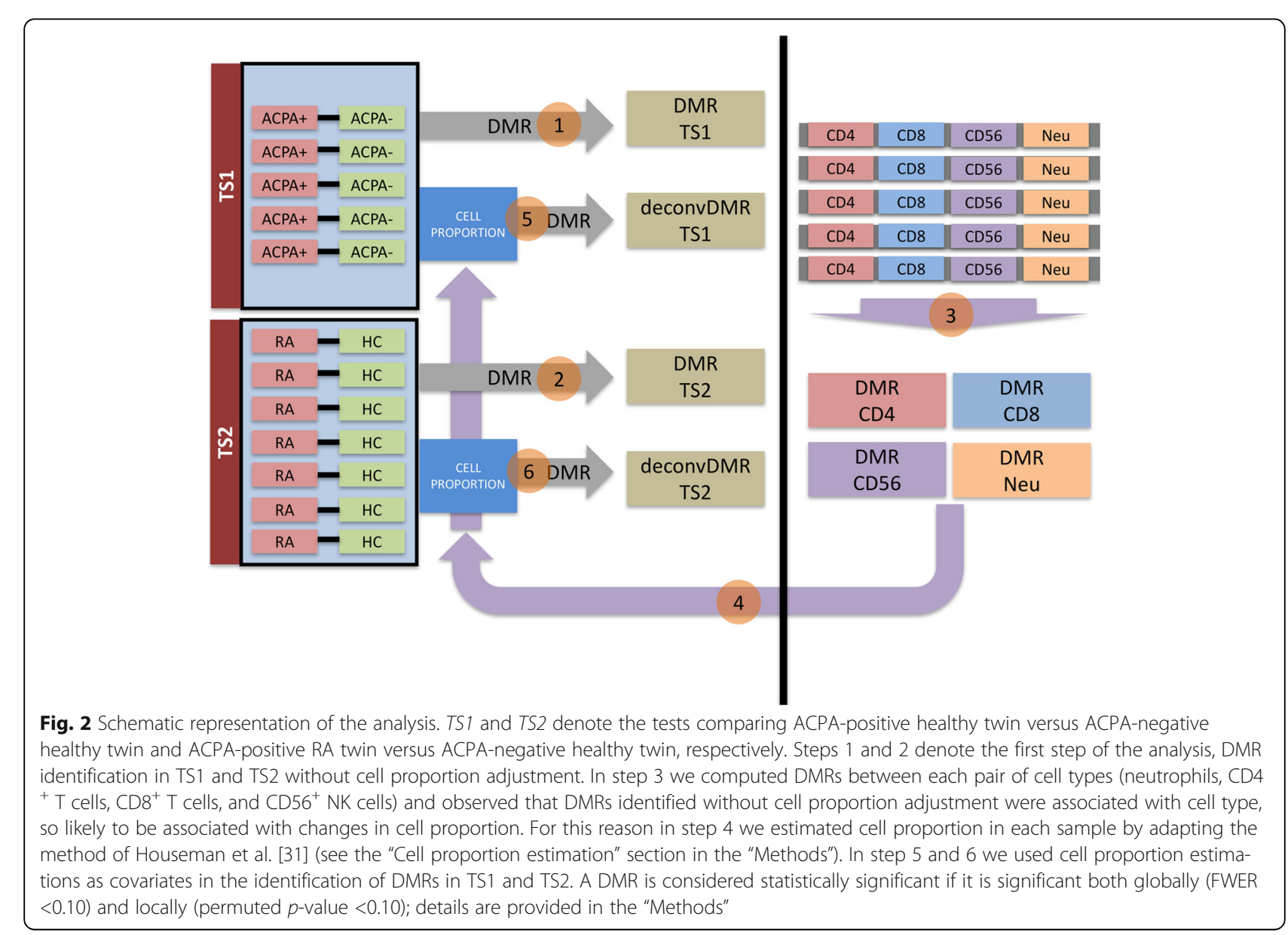


Table 2 Estimation of cell proportion

\begin{tabular}{|c|c|c|c|c|c|c|c|c|c|c|c|c|}
\hline \multirow[t]{2}{*}{ PAIR } & \multicolumn{4}{|c|}{ ACPA titer positive and healthy, $\%$} & \multicolumn{4}{|c|}{ ACPA titer negative and healthy, $\%$} & \multicolumn{4}{|c|}{ DIF } \\
\hline & $\begin{array}{c}\text { Neutrop } \\
\text { hils }\end{array}$ & $\begin{array}{l}\text { CD4+ T } \\
\text { cells }\end{array}$ & $\begin{array}{l}\text { CD8+ T } \\
\text { cells }\end{array}$ & $\begin{array}{c}\text { CD56+ NK } \\
\text { cells }\end{array}$ & $\begin{array}{l}\text { Neutrop } \\
\text { hils }\end{array}$ & $\begin{array}{l}\text { CD4+ T } \\
\text { cells }\end{array}$ & $\begin{array}{l}\text { CD8+T } \\
\text { cells }\end{array}$ & $\begin{array}{c}\text { CD56+ NK } \\
\text { cells }\end{array}$ & $\begin{array}{l}\text { Neutrop } \\
\text { hils }\end{array}$ & $\begin{array}{l}\text { CD4+ T } \\
\text { cells }\end{array}$ & $\begin{array}{l}\text { CD8+T } \\
\text { cells }\end{array}$ & $\begin{array}{c}\text { CD56+ NK } \\
\text { cells }\end{array}$ \\
\hline PAIR_1 & 0.43 & 0.15 & 0.36 & 0.07 & 0.58 & 0.14 & 0.17 & 0.10 & -0.16 & 0.01 & 0.18 & -0.03 \\
\hline PAIR_2 & 0.52 & 0.05 & 0.22 & 0.21 & 0.78 & 0.11 & 0.06 & 0.06 & -0.26 & -0.05 & 0.16 & 0.15 \\
\hline PAIR_3 & 0.44 & 0.39 & 0.17 & 0.00 & 0.43 & 0.26 & 0.24 & 0.07 & 0.01 & 0.14 & -0.07 & -0.07 \\
\hline PAIR_4 & 0.51 & 0.22 & 0.15 & 0.12 & 0.44 & 0.26 & 0.25 & 0.04 & 0.07 & -0.05 & -0.10 & 0.08 \\
\hline PAIR_5 & 0.62 & 0.08 & 0.23 & 0.06 & 0.45 & 0.11 & 0.44 & 0.00 & 0.17 & -0.03 & -0.21 & 0.06 \\
\hline & & & & & & & & $\mathrm{t}$-test & 0.69 & 0.94 & 0.93 & 0.40 \\
\hline
\end{tabular}

\begin{tabular}{|c|c|c|c|c|c|c|c|c|c|c|c|c|}
\hline \multirow[t]{2}{*}{$\begin{array}{l}\text { TS2 } \\
\text { PAIR }\end{array}$} & \multicolumn{4}{|c|}{ RA and ACPA titer positive, $\%$} & \multicolumn{4}{|c|}{ ACPA titer negative healthy individual, $\%$} & \multicolumn{4}{|c|}{ DIF } \\
\hline & $\begin{array}{c}\text { Neutrop } \\
\text { hils }\end{array}$ & $\begin{array}{l}\text { CD4+ T } \\
\text { cells }\end{array}$ & $\begin{array}{l}\text { CD8+ T } \\
\text { cells }\end{array}$ & $\begin{array}{c}\text { CD56+ NK } \\
\text { cells }\end{array}$ & $\begin{array}{c}\text { Neutrop } \\
\text { hils }\end{array}$ & $\begin{array}{l}\text { CD4+T } \\
\text { cells }\end{array}$ & $\begin{array}{l}\text { CD8+ T } \\
\text { cells }\end{array}$ & $\begin{array}{c}\text { CD56+ NK } \\
\text { cells }\end{array}$ & $\begin{array}{c}\text { Neutrop } \\
\text { hils }\end{array}$ & $\begin{array}{l}\text { CD4+ T } \\
\text { cells }\end{array}$ & $\begin{array}{l}\text { CD8+ T } \\
\text { cells }\end{array}$ & $\begin{array}{c}\text { CD56+ NK } \\
\text { cells }\end{array}$ \\
\hline PAIR_1 & 0.65 & 0.23 & 0.03 & 0.08 & 0.63 & 0.15 & 0.10 & 0.11 & 0.02 & 0.08 & -0.07 & -0.03 \\
\hline PAIR_2 & 0.60 & 0.10 & 0.23 & 0.08 & 0.66 & 0.19 & 0.05 & 0.11 & -0.06 & -0.09 & 0.18 & -0.03 \\
\hline PAIR_3 & 0.67 & 0.12 & 0.13 & 0.08 & 0.54 & 0.20 & 0.13 & 0.13 & 0.13 & -0.08 & 0.00 & -0.05 \\
\hline PAIR_4 & 0.61 & 0.20 & 0.07 & 0.12 & 0.66 & 0.21 & 0.05 & 0.08 & -0.05 & -0.01 & 0.02 & 0.04 \\
\hline PAIR_5 & 0.51 & 0.33 & 0.17 & 0.00 & 0.45 & 0.28 & 0.23 & 0.03 & 0.05 & 0.05 & -0.07 & -0.03 \\
\hline PAIR_6 & 0.72 & 0.18 & 0.09 & 0.01 & 0.53 & 0.26 & 0.12 & 0.10 & 0.19 & -0.08 & -0.03 & -0.09 \\
\hline PAIR_7 & 0.58 & 0.25 & 0.12 & 0.04 & 0.50 & 0.25 & 0.15 & 0.10 & 0.09 & 0.01 & -0.03 & -0.06 \\
\hline & & & & & & & & t-test & 0.18 & 0.51 & 1.00 & 0.06 \\
\hline
\end{tabular}

For each sample the estimation of neutrophils, CD4 ${ }^{+} \mathrm{T}$ cells, CD8 ${ }^{+} \mathrm{T}$ cells, and $\mathrm{CD} 56^{+} \mathrm{NK}$ cells is provided, as described by Houseman et al. [31]. For each twin pair the difference in cell proportion (computed in ratios from 0 to 1, equivalent to percentages) for each cell type was computed in DIF; the purple cells indicate the $p$ values from the paired $t$-test comparing differences. The sum of all ratios for a sample may differ from 1 due to rounding in the table; the analysis was performed without rounding

Table 3 DMRs identified after cell type correction

\begin{tabular}{|c|c|c|c|c|c|c|c|c|c|}
\hline$\overline{\text { DMR name }}$ & Chromosome & Start & End & nprobes & FWER average & FWER maximum & FWER area & Permuted $p$ value & Type \\
\hline$\overline{\text { DMR1 }}$ & Chr5 & 140582954 & 140584018 & 31 & $<0.01$ & $<0.05$ & $<0.005$ & 0.06 & TS1 \\
\hline DMR2 & Chr11 & 74630937 & 74631216 & 7 & $<0.05$ & $<0.01$ & $<0.1$ & 0.03 & TS1 \\
\hline DMR3 & ChrX & 51087402 & 51089195 & 32 & $<0.1$ & $<0.1$ & $<0.01$ & 0.03 & TS1 \\
\hline DMR4 & Chr14 & 54101669 & 54102157 & 6 & $<0.1$ & $<0.1$ & & 0.03 & TS1 \\
\hline DMR5 & Chr2 & 85215637 & 85215811 & 6 & $<0.1$ & & & 0.06 & TS1 \\
\hline DMR6 & Chr14 & 96038274 & 96038760 & 11 & & $<0.01$ & & 0.06 & TS1 \\
\hline DMR7 & Chr9 & 4731364 & 4731640 & 6 & & $<0.05$ & & 0.03 & TS1 \\
\hline DMR8 & Chr1 & 198645284 & 198645642 & 7 & & $<0.1$ & & 0.03 & TS1 \\
\hline DMR9 & Chr1 & 116184060 & 116185215 & 33 & & & $<0.05$ & 0.06 & TS1 \\
\hline DMR10 & Chr15 & 75897924 & 75898101 & 6 & & $<0.05$ & & 0.01 & TS1 \\
\hline DMR11 & Chr2 & 171280327 & 171280711 & 10 & $<0.1$ & & & 0.03 & TS1 \\
\hline DMR12 & Chr16 & 66835947 & 66836364 & 12 & & $<0.05$ & & 0.09 & TS1 \\
\hline DMR13 & Chr13 & 113192663 & 113193262 & 8 & & $<0.1$ & & 0.06 & TS1 \\
\hline DMR14 & Chr22 & 19603181 & 19604444 & 33 & & & $<0.1$ & 0.06 & TS1 \\
\hline DMR15 & Chr17 & 41620864 & 41621503 & 16 & & & $<0.1$ & 0.06 & TS1 \\
\hline DMR16 & Chr8 & 61353541 & 61354034 & 15 & & & $<0.1$ & 0.06 & TS1 \\
\hline DMR17 & ChrX & 113720992 & 113721797 & 22 & & & $<0.1$ & 0.06 & TS1 \\
\hline DMR18 & Chr10 & 99200020 & 99200806 & 23 & $<0.05$ & $<0.05$ & $<0.005$ & $<0.01$ & TS2 \\
\hline
\end{tabular}

DMRs (differentially methylated probes) identified after cell type correction in TS1 and TS2; the double criteria for selection is first a FWER <0.20 in at least one of the three statistics (italics) and secondly a permuted $p$ value <0.1. "Start" and "End" denote the start and end location of the DMR in the chromosome. "nprobes" denotes the number of probes in the DMR. "FWER average", "FWER maximum", and "FWER area" denote the FWER associated with each one of the statistics used (see "Methods"). "Permuted $p$ value" denotes the $p$ value computed for each DMR locally, comparing each DMR statistic to random permutations of the samples. "Type" denotes if the DMR is associated with TS1 (ACPA/healthy discordant) or TS2 (ACPA-positive RA/healthy discordant). Bold highlights DMRs whose FWER was $<0.005$. Chromosomal locations are based on the hg18 build 
Table 4 Characterization of DMRs identified after cell type correction

\begin{tabular}{|c|c|c|c|c|c|}
\hline$\overline{\text { DMR name }}$ & Gene (distance to TSS) & Projection & Location: gene & Location: CGI & EIRA \\
\hline$\overline{\mathrm{DMR} 1}$ & PCDHB14 (+224) & 0.02 & Promoter & Shore & No \\
\hline DMR2 & SLCO2B1 (+91266), ARRB1 (+109444) & 0.28 & Intergenic & CGl/Shore & $Y_{e s}^{a}$ \\
\hline DMR3 & NUDT10 (-3524) & 0.48 & Gene body & - & No \\
\hline DMR4 & SAMD4A (-2474) & 0.67 & Gene body & CGl & Yes + \\
\hline DMR5 & TCF7L1 (+1479), TGOLN2 (+193161) & 0.30 & Promoter & CGl & $\mathrm{Yes}^{\mathrm{a}}+$ \\
\hline DMR6 & PAPOLA (+44) & 0.56 & Promoter & CGl & Yes $^{a}+$ \\
\hline DMR7 & AK3 $(-275)$ & 0.84 & Promoter & CGl & Yes + \\
\hline DMR8 & ZNF281 (+326) & 0.89 & Promoter & CGl & Yes + \\
\hline DMR9 & NHLH2 (+632) & 0.55 & Promoter & Shore & Yes \\
\hline DMR10 & LINGO1 (-186249), TBC1D2B (+259036) & 0.45 & Intergenic & CGI/Shore & No \\
\hline DMR11 & SP5 (+412) & 0.83 & Promoter & CGl & Yes + \\
\hline DMR12 & PLA2G15 (-592) & 0.31 & Promoter & CGl/Shore & Yes + \\
\hline DMR13 & TMCO3 $(-346)$ & 0.73 & Promoter & CGI & Yes + \\
\hline DMR14 & LZTR1 (-62745), CRKL (+2099) & 0.13 & Extended promoter & Shore & * \\
\hline DMR15 & LRRC37A (-107090), KIAA1267 (-15809) & 0.94 & Gene body & - & * \\
\hline DMR16 & CA8 $(+2720)$ & 0.91 & Extended promoter & - & * \\
\hline DMR17 & HTR2C (-3412) & 0.20 & Gene body & Shore & * \\
\hline DMR18 & EXOSC1 (-4655), ZDHHC16 (+4493) & 0.69 & Gene body & Shore & No \\
\hline
\end{tabular}

"Gene" provides information on the closest genes and the distance to the transcription start site (TSS). "Location: gene" and "Location: CGI" denote the locations of the center of the DMR in relation to a gene or a CpG island. "EIRA" is "yes" if the DMR overlaps with a DMP from the EIRA cohort [2] or if it is "located within $1000 \mathrm{bp}$ of one; a plus sign in the EIRA column denotes the same direction of change. "Projection" denotes the permuted $p$ value of the DMRs of TS1 and TS2 when using methylation data from TS2 and TS1, respectively (see the "Projection analysis" section in the "Methods"); $p$ values in bold are those $<0.05$. An asterisk denotes that no probes within $500 \mathrm{bp}$ of the DMR are available in the Illumina $450 \mathrm{~K}$ array and no comparison is thus possible

overlapping DMRs are located within 200 bp of the differentially methylated probes identified after cell correction in our previous study of drug-naïve ACPA-positive RA (the EIRA cohort) using Illumina $450 \mathrm{~K}$ analysis [2]. Furthermore, the changes in EIRA were directionally consistent with changes in CHARM analysis at eight of the ten sites. The EIRA cohort is a Swedish populationbased case-control study (for more information on this cohort, please visit http://www.eirasweden.se/index1.htm).

One DMR (DMR18; Table 3; Additional file 1: Figure S23) was found when analyzing the TS2 group with cell type proportion correction (FWER $\leq 0.10$ ). DMR18 spans 23 consecutive probes and is associated with the genes EXOSC1 and ZDHHC16 (Tables 3 and 4; Additional file 1: Figure S23). This DMR is hypermethylated in ACPApositive RA and located in a CpG island shore. ZDHHC16 is a probable palmitoyltransferase gene and the EXOSC1 gene codes for a core component of the exosome, highly pertinent for innate immunity. Several proteins of this complex are targets of autoantibodies in patients with autoimmune disease [39]. Interestingly, EXOSC1 is also a significant DMR in the analysis before adjustment for cell type (Additional file 2: Table S2). The DMR18 was not identified in the EIRA study after cell type adjustment.
Additionally, a cell correction-based technical replication was conducted for the bisulfite pyrosequencing by comparing profiles generated previously for PCDHB14 (for TS1) and EXOSC1 (for TS2) with the cell proportion estimated by CHARM (see the "Statistical analysis for validation" in the "Methods"); in all cases the change is directionally consistent but without statistical significance (Additional file 2: Table S4, marked in orange).

\section{Analysis of TS1 candidate differentially methylated regions in the TS2 group}

While cell type correction is necessary for a mechanistic understanding of gene regulation and disease pathology (Additional file 1: Figure S6) [2], the methodology to estimate cell proportions introduces other types of technical variance. Since we have a limited number of paired twins in TS1 and TS2 but larger variation in cell proportion estimates in the disease discordant TS2 group, we hypothesize that cell proportion correction methodology may introduce larger variance in TS2 than in TS1, and therefore we have lower power to identify DMRs (despite higher absolute log-transformed fold change in TS2). Hence, this may be the reason for the limited overlap between TS1 and TS2 after cell type deconvolution. 


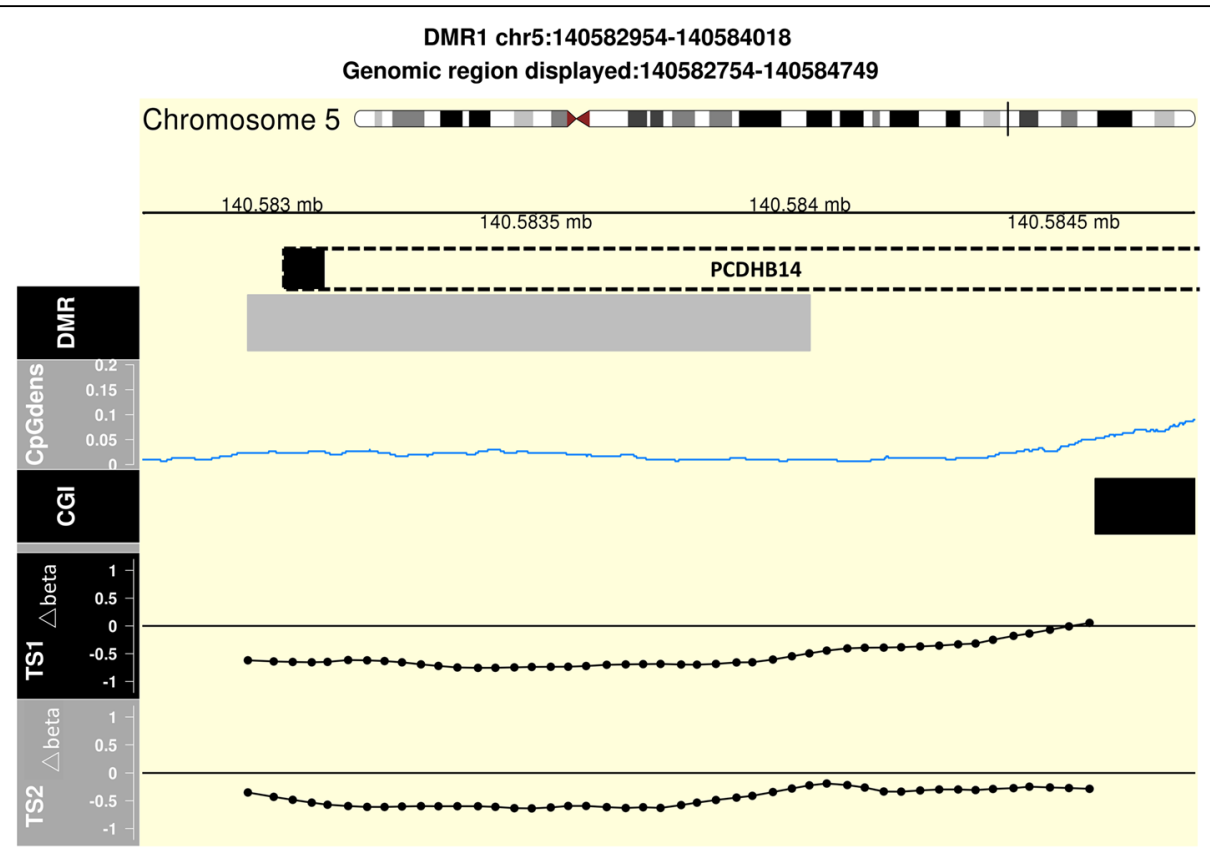

Fig. 3 DMR1, from TS1 after cell proportion correction, at the promoter region of PCDHB14 (chr5 140582954-140584018). DMR denotes the DMR location (grey box); CpGdens denotes CpG density as computed by CHARM [27] (CpG); CGI denotes the location of CpG islands (black box). TS1 $\triangle$ beta and TS2 $\Delta$ beta shows the smoothed linear slope (differences in methylation or delta) associated with ACPA-positive healthy and ACPA-positive RA twin, respectively, in the linear model which is used CHARM [27] to identify DMR candidates. Every point denotes a probe location. The location of the gene PCDHB14 is shown in a dotted box; the black square on the left denotes the location of the transcription start site

To test this hypothesis and to investigate the possible relationship between the two different phases of disease development, we investigated if TS1 DMRs are significant based on permuted $p$ values in the TS2 analysis. A single DMR (DMR1) discovered in the cell type-adjusted healthy ACPA discordant analysis (TS1) is statistically significant in ACPA-positive RA data (TS2) (see the "Projection analysis" section in the "Methods"; indicated in bold in Table 4). DMR1 is located in the promoter of PCDHB14, part of the protocadherin beta gene cluster [40]. Additionally and importantly, we observed no TS2 DMR that was significant in the TS1 analysis.

\section{Discussion}

The presence of ACPAs preceding the RA phenotype in conjunction with accessible clinical samples supports the notion of utilizing the pathology of ACPA-positive $\mathrm{RA}$ as an autoimmunity disease prototype, facilitating the temporal analysis of the contributions of epigenetic modifications in the context of genes and environment. The current study was therefore designed to elucidate epigenetic factors, albeit not functionally causal, associated with ACPA and the development of ACPA-positive RA that are not directly caused by genetic contributions. The pathogenesis of ACPA-positive RA has strong genetic associations in both the MHC cluster on chromosome
6, where the $H L A$ genes of the adaptive immune system reside, as well as non-immune genes. A wealth of information for RA has come from comprehensive genome-wide association studies, and from this it has even been possible to determine the involved individual amino acids in functional domains for antigen presentation [41]. We have previously analyzed how epigenetic factors integrate with genotype by employing DNA methylation profiling in an ACPA-positive RA case-control study [2]. In this way, novel genetic associations with genes were revealed that also associated with specific patterns of DNA methylation. It is also important, however, to separate components of the etiology and pathogenesis of RA from the genetic background. Our results also suggest that the twin approach employed here is useful to neutralize the genetic components. Thus, we did not find any DMRs in the MHC region in the current study, likely due to such neutralization of genetic differences in the discordant $\mathrm{MZ}$ twins, supporting the notion that differential methylation in the MHC cluster in RA may actually be completely driven by the genotype. Furthermore, none of the loci in the over 100 previously known genotype-dependent non-MHC genes overlaps with our identified DMRs [21]. One of these genes, however, IRF5, with known genotype associations with RA, contains a DMR in the nondeconvoluted TS2 group, although no known associated 
SNPs are in this region. Since this gene has many associated polymorphisms, this may suggest a genetic-epigenetic interaction, which is worth further investigation as discussed below.

Our findings reveal an enrichment towards probe hypomethylation in ACPA-positive RA ( $p$ value $<10 \mathrm{e}(-9)$ ), in line with Karouzkis et al. [42] and Liu et al. [2]. The CHARM methodology has, however, not previously been used to analyze the RA methylome. Importantly, the CHARM design enables an optimized estimation of DMRs defined by close consecutive probes targeting methylated regions rather than single $\mathrm{CpG}$ positions [22]. In addition, DMR methodologies have lower power when applied to the Infinium Illumina $450 \mathrm{~K}$ array because the latter design includes many isolated probes yielding DMPs. Furthermore, the CHARM array used here employs a 2.1 million feature array compared to the 480,000 probes (and CpGs) in the Infinium Illumina $450 \mathrm{~K}$ array and the recent Infinium Methylation EPIC bead array covering 850,000 probes [43]. A region of differentially methylated CpGs is also a stronger and more robust indicator of altered methylation compared to single CpGs; however, methodologies for DMR power analysis are not yet available while there are methodologies for DMP power estimation $[26,36]$.

Furthermore, since the cell type distribution in the whole blood cell population was likely to differ between the healthy and affected twin within the pairs, a deconvolution algorithm was applied for CHARM, based on known cell type-specific methylation profiles from four cell types, $\mathrm{CD}^{+}$and $\mathrm{CD}^{+}{ }^{+} \mathrm{T}$ lymphocytes, $\mathrm{CD} 56^{+} \mathrm{NK}$ cells, and neutrophils. The deconvolution yields an estimate of the relative distribution of these cell types in order to avoid the discovery of methylation pattern changes driven mostly by changes in differential cell count. It is important to realize that when analyzing non-deconvoluted data, the apparent lack of disease-specific differential methylation in a particular CpG may be due to one disease-affected cell type with hypermethylation in that site and another cell type without this change counteracting the overall methylation alteration. This may create a false negative result. Our results clearly reveal that the analysis of deconvoluted data and unadjusted data answer different questions and show the importance of adjusting for the cell type composition when approaching actual (non-cell type-driven) epigenetic changes within a heterogeneous cell population. However, phenotypic changes of cell type characteristics, regarding methylation, may be partially lost by the deconvolution. In addition, the view of a disease-specific cell population may be valuable for biomarker discovery. In this analysis we cannot interpret whether this differential methylation is due to increases in a certain cell type or a change of cell type distribution in parallel with methylation changes. We did not find significant functional enrichment for the identified genes (see the "Functional analyses" section in the "Methods").

The current study aimed to reveal novel regions and genes involved in the temporal development of ACPApositive RA. By including the ACPA-positive discordant healthy twin pair group, we were able to compare two distinct phases in the development of ACPA-positive RA. The twin set discordant for ACPA should be considered as healthy but at increased risk for developing ACPA-positive RA. The exact risk of developing RA is not known and some twins might never develop RA due to additional protective factors or random factors, while others will. Considering the low power observed in TS2, we performed a targeted analysis to investigate if any genomic regions identified in TS1 (TS1 DMRs) were significant in TS2 using TS2 data (see the "Projection analysis" section in the "Methods"). Interestingly, the top DMR (DMR1 in Table 3) discovered in the deconvoluted healthy ACPA-discordant analysis (TS1) was identified as a relevant candidate also in ACPA-positive RA data (TS2) ( $p$ value $=0.02$ ). This overlapping DMR1 is associated with the PCDHB14 gene. The overlap could imply the involvement of the associated genes in an ACPA-positive RA disease trajectory. In our analysis, several DMRs associate with $P C D H$ genes, both in TS1 and TS2. The relevance of $P C D H$ genes in ACPA and RA phenotypes is further emphasized by the significant outcome from the meta analysis of the bisulfite pyrosequencing. The protocadherin family, with over 70 identified genes, are members of the cadherin super family. They are divided into over 50 clustered $\alpha$-, $\beta$-, and $\gamma-P C D H$ genes, all located on chromosome 5 , and non-clustered genes scattered in the genome. These transmembrane protein genes are differentially expressed, predominantly in neuronal dendrites, and have been reported to be involved in self/non-self-recognition and self-avoidance [44]. Furthermore, the PCDH18 protein was recently reported as an activation marker of $\mathrm{CD}^{+}$memory $\mathrm{T}$ cells [45]. The $P C D H$ gene clusters have a genomic organization similar to B-cell and T-cell receptor gene clusters. This, together with their differential methylation pattern identified in TS1 and TS2, could imply a role in self-recognition and autoimmunity.

The one significant DMR after adjustment for cell type from the ACPA-positive RA discordant twins associates with the EXOSC1 gene, which codes for a core component of the exosome involved in the processing, controlling, and degrading of RNA and in cytokine regulation and autoimmunity [46]. The exosome has also been shown to be important in the creation of immunoglobulin diversification [47]. Autoantibodies directed towards components of the exosome complex have been identified in sera of patients with idiopathic inflammatory myopathy (IIM), scleroderma, and PM/Scl overlap syndrome 
[48]. Also, bioinformatic analysis targeting inflammatory bowel disease (IBD), among other diseases, revealed that EXOSC1 was one of the top upregulated genes associated with the disease [49]. The EXOSC1 gene deserves further attention and the role of epigenetically regulated gene regions such as enhancers should be investigated. Since the EXOSC1 DMR was not identified in the healthy ACPA discordant group, it may be specific to fully developed RA rather than a pre-stage. This DMR was not found to replicate the previous non-twin EIRA study (in which the ACPA-positive RA patients were drug-naïve); therefore, we cannot exclude the possibility that it is driven by DMARD therapy (Additional file 2: Table S1). The number of genes which to any extent associated with differential methylation within the pairs in the RA discordant TS2 group are substantially less (and not all overlapping) compared with those found in the EIRA study. Various reasons for this may exist, but at least two main differences stand out regarding experimental design. First and considering the number of samples, our study has lower statistical power than in the EIRA study. Second, the previous study interrogated DMPs using the Illumina $450 \mathrm{~K}$ platform and this study employed CHARM to investigate DMRs, and in addition the probes have limited overlap (as shown in our plots of cDMRs). Third, the current study neutralizes any genetic influence on the differential methylation of RA versus non-RA, which the previous study employing a genetically heterogeneous cohort did not do; therefore, we may expect a limited overlap. Finally, the EIRA study comprises treatment-naïve RA patients, again possibly implying an effect of DMARDs in the current study.

Although novel associations of DNA methylation have been implicated in phases of development of ACPApositive RA, we cannot at this time determine whether this is causally dependent on DNA methylation. To investigate possible confounders for each DMR, we looked into potential associations between changes in DNA methylation with the following covariates: gender, age, smoking, and HLA epitope information (Additional file 2: Table S3). No significant associations were found ( $p$ value $<0.01$ ), although the results showed that age (for TS1) and gender (for TS2) are covariates to be investigated further in larger cohorts. Importantly, our main candidate, $P C D H B 14$, is not significantly associated with any covariate.

Our findings do support the notion that DMR1 from the ACPA-positive healthy individuals analysis (and associated with PCDHB14) may be associated with onset of ACPA-positive RA, since this DMR could also be found when analyzed in the context of twin pairs discordant for ACPA-positive RA. The discovered genes associated with DMRs found here can be further used for hypothesis generation.

\section{Conclusions}

Here we used a general statistical framework, adapted to empower a low-sample twin design. This new robust framework was applied to the DNA methylome from two small sets of MZ twins discordant for ACPAs but healthy and ACPA-positive RA, respectively. The unique material for the data represents different phases during the progression of RA, thus enabling us for the first time to interrogate the temporal contribution of epigenetic factors dissociated from genetics to the evolution of the disease. This design made it possible to delineate candidate genes of relevance for development of ACPApositive RA. The DMR associated with a $P C D H$ gene suggests a temporal epigenetic connection between ACPA-positivity and clinical RA. Our results should be of interest for further research in the clinical autoimmune field for hypothesis generation, as well as for the wider research community employing the proposed statistical approach.

\section{Additional files}

Additional file 1: Supplementary Figures S1 to S26. (ZIP $7 \mathrm{mb}$ )

Additional file 2: Supplementary Tables S1 to S4. (ZIP $31 \mathrm{~kb}$ )

Additional file 3: Supplementary methods. The file includes extended description of aspects of the bioinformatic analysis. (DOCX $25 \mathrm{~kb}$ )

\section{Funding}

This work was financed by AFA Insurance, the Swedish Research Council, the Swedish Rheumatism Association, and the Swedish Research Foundation of Strategic Research (RBc08-0027), and the STATegra EU FP7 project (306000) The funding bodies have no role in the design of the study, the collection, analysis, or interpretation of data, or in writing the manuscript.

\section{Availability of data and materials}

Microarray datasets generated and/or analyzed during the current study are available in NCBI's Gene Expression Omnibus (GEO) [50] and are accessible through GEO Series accession numbers GSE72582 (http:// www.ncbi.nlm.nih.gov/geo/query/acc.cgi?acc=GSE72582) and GSE72586 (http://www.ncbi.nlm.nih.gov/geo/query/acc.cgi?acc=GSE72586).

Non-microarray datasets in the current study (e.g., pyrosequencing data) are available from the corresponding author on request (tomas.ekstrom@ki.se). The publicly available data discussed in the publication are available in NCBI's Gene Expression Omnibus [50] and are accessible through GEO Series accession number GSE42861 (http://www.ncbi.nlm.nih.gov/geo/query/ acc.cgi?acc=GSE42861); these data were originally discussed in [2].

\section{Authors' contributions}

DG-C participated in project planning and performed the bioinformatic computations, interpreted results, wrote the manuscript, and prepared figures; MA and LKS participated in the project planning, performed CHARM experimental work, performed validation by bisulfite pyrosequencing, interpreted results, and wrote the manuscript; $\mathrm{AHH}$ selected all samples, performed immunological analyses, validated clinical diagnoses, and wrote the manuscript; MVR performed validations and replications by bisulfite pyrosequencing and reviewed the manuscript; RT performed CHARM analysis; JK, AS, NA, and LR collected blood and isolated different cell types from healthy controls for the cell type adjustments; MJA, MAT, and CM contributed bioinformatic expertise and reviewed the biostatistical analysis; JIF performed CHARM of the sorted control cell types; APF participated in planning and execution of the analyses and the interpretation of the data; JT, LK, and AC contributed in the planning of the project and analytical expertise; TJE conceived 
and coordinated the project, planned the experiments, and wrote the manuscript. All authors have participated in correction and proofreading of the manuscript.

\section{Competing interests}

The authors declare that they have no competing interests.

\section{Ethics approval and consent to participate}

Each individual has given written approval for participation in the study and the ethical review board at Karolinska Institutet has approved the study.

\section{Author details}

${ }^{1}$ Center for Molecular Medicine at Karolinska Institutet and Karolinska University Hospital, Stockholm, Sweden. ${ }^{2}$ Department of Medicine, Unit of Computational Medicine, Stockholm, Sweden. ${ }^{3}$ Bioinformatic Infrastructure for Life Sciences, Stockholm, Sweden. ${ }^{4}$ Department of Clinical Neuroscience, Karolinska Institutet, Stockholm, Sweden. ${ }^{5}$ Department of Medicine, Unit of Rheumatology, Karolinska University Hospital Solna, Stockholm, Sweden. ${ }^{6}$ Center for Biosciences, Department of Biosciences and Nutrition, Karolinska Institutet, Stockholm, Sweden. ${ }^{7}$ Translational Immunology Unit, Department of Medicine Solna, Karolinska Institutet and Karolinska University Hospital, Stockholm, Sweden. ${ }^{8}$ Center for Epigenetics, Johns Hopkins University, Baltimore, MD, USA. ${ }^{9}$ Department of Medicine, Johns Hopkins University, Baltimore, MD, USA. ${ }^{10}$ Departments of Biostatistics, Johns Hopkins University, Baltimore, MD, USA. ${ }^{11}$ Departments of Mental Health, Bloomberg School of Public Health, Johns Hopkins University, Baltimore, MD, USA. ${ }^{12}$ Medical Scientist Training Program, and Predoctoral Training Program in Human Genetics, McKusick-Nathans Institute of Genetic Medicine, Johns Hopkins University School of Medicine, Baltimore, MD, USA. ${ }^{13}$ Departments of Pathology, Massachusetts General Hospital, Charlestown, MA, USA. ${ }^{14}$ Harvard Medical School, Boston, MD, USA. ${ }^{15}$ Biostatistics, Harvard TH Chan School of Public Health, Boston, MA, USA. ${ }^{16}$ Broad Institute of Harvard and MIT, Cambridge, MA, USA. ${ }^{17}$ Mucosal and Salivary Biology Division, King's College London Dental Institute, London, UK. ${ }^{18}$ Department of Clinical Science and Education, Karolinska Institutet, and Sachs' Children and Youth Hospital, Södersjukhuset, Stockholm, Sweden.

Received: 4 October 2016 Accepted: 20 October 2016 Published online: 22 November 2016

\section{References}

1. Feinberg AP. Phenotypic plasticity and the epigenetics of human disease. Nature. 2007:447:433-40.

2. Liu Y, Aryee MJ, Padyukov L, Fallin MD, Hesselberg E, Runarsson A, Reinius L, Acevedo N, Taub M, Ronninger M, et al. Epigenome-wide association data implicate DNA methylation as an intermediary of genetic risk in rheumatoid arthritis. Nat Biotechnol. 2013;31:142-7 (http://www.ncbi.nlm.nih.gov/geo/ query/acc.cgi?acc=GSE42861).

3. Marsit CJ. Influence of environmental exposure on human epigenetic regulation. J Exp Biol. 2015;218:71-9.

4. Irizarry RA, Ladd-Acosta C, Wen B, Wu Z, Montano C, Onyango P, Cui H, Gabo K, Rongione M, Webster M, et al. The human colon cancer methylome shows similar hypo- and hypermethylation at conserved tissue-specific CpG island shores. Nat Genet. 2009:41:178-86.

5. Ziller MJ, Gu H, Muller F, Donaghey J, Tsai LT, Kohlbacher O, De Jager PL, Rosen ED, Bennett DA, Bernstein BE, et al. Charting a dynamic DNA methylation landscape of the human genome. Nature. 2013;500:477-81.

6. Neovius M, Simard JF, Askling J. Nationwide prevalence of rheumatoid arthritis and penetration of disease-modifying drugs in Sweden. Ann Rheum Dis. 2011;70:624-9.

7. Catrina Al, Ytterberg AJ, Reynisdottir G, Malmstrom V, Klareskog L. Lungs, joints and immunity against citrullinated proteins in rheumatoid arthritis. Nat Rev Rheumatol. 2014;10:645-53.

8. Klareskog L, Catrina Al, Paget S. Rheumatoid arthritis. Lancet. 2009;373:659-72.

9. Nielen MM, van Schaardenburg $D$, Reesink HW, van de Stadt RJ, van der Horst-Bruinsma IE, de Koning MH, Habibuw MR, Vandenbroucke JP, Dijkmans BA. Specific autoantibodies precede the symptoms of rheumatoid arthritis: a study of serial measurements in blood donors. Arthritis Rheum. 2004;50:380-6.

10. Rantapaa-Dahlqvist S, de Jong BA, Berglin E, Hallmans G, Wadell G, Stenlund $H$, Sundin U, van Venrooij WJ. Antibodies against cyclic citrullinated peptide and IgA rheumatoid factor predict the development of rheumatoid arthritis. Arthritis Rheum. 2003;48:2741-9.

11. Hensvold AH, Magnusson PK, Joshua V, Hansson M, Israelsson L, Ferreira R, Jakobsson PJ, Holmdahl R, Hammarstrom L, Malmstrom V, et al. Environmental and genetic factors in the development of anticitrullinated protein antibodies (ACPAs) and ACPA-positive rheumatoid arthritis: an epidemiological investigation in twins. Ann Rheum Dis. 2015;74:375-80.

12. van de Stadt $L A$, van der Horst $A R$, de Koning $M H$, Bos WH, Wolbink GJ, van de Stadt RJ, Pruijn GJ, Dijkmans BA, van Schaardenburg D, Hamann D. The extent of the anti-citrullinated protein antibody repertoire is associated with arthritis development in patients with seropositive arthralgia. Ann Rheum Dis. 2011;70:128-33.

13. de Rooy DP, van der Linden MP, Knevel R, Huizinga TW, van der Helm-van Mil AH. Predicting arthritis outcomes-what can be learned from the Leiden Early Arthritis Clinic? Rheumatology (Oxford). 2011;50:93-100.

14. Gerlag DM, Raza K, van Baarsen LG, Brouwer E, Buckley CD, Burmester GR, Gabay C, Catrina Al, Cope AP, Cornelis F, et al. EULAR recommendations for terminology and research in individuals at risk of rheumatoid arthritis: report from the Study Group for Risk Factors for Rheumatoid Arthritis. Ann Rheum Dis. 2012;71:638-41.

15. Brink M, Hansson M, Mathsson L, Jakobsson PJ, Holmdahl R, Hallmans G, Stenlund H, Ronnelid J, Klareskog L, Rantapaa-Dahlqvist S. Multiplex analyses of antibodies against citrullinated peptides in individuals prior to development of rheumatoid arthritis. Arthritis Rheum. 2013;65:899-910.

16. Strickland FM, Richardson BC. Epigenetics in human autoimmunity. Epigenetics in autoimmunity-DNA methylation in systemic lupus erythematosus and beyond. Autoimmunity. 2008;41:278-86.

17. Klareskog L, Stolt P, Lundberg K, Kallberg H, Bengtsson C, Grunewald J, Ronnelid J, Harris HE, Ulfgren AK, Rantapaa-Dahlqvist S, et al. A new model for an etiology of rheumatoid arthritis: smoking may trigger HLA-DR (shared epitope)-restricted immune reactions to autoantigens modified by citrullination. Arthritis Rheum. 2006:54:38-46.

18. Karlson EW, Chang SC, Cui J, Chibnik LB, Fraser PA, De Vivo I, Costenbader $\mathrm{KH}$. Gene-environment interaction between HLA-DRB1 shared epitope and heavy cigarette smoking in predicting incident rheumatoid arthritis. Ann Rheum Dis. 2010;69:54-60.

19. Frisell T, Holmqvist M, Kallberg H, Klareskog L, Alfredsson L, Askling J. Familial risks and heritability of rheumatoid arthritis: role of rheumatoid factor/anti-citrullinated protein antibody status, number and type of affected relatives, sex, and age. Arthritis Rheum. 2013;65:2773-82.

20. Cui J, Taylor KE, Lee YC, Kallberg H, Weinblatt ME, Coblyn JS, Klareskog L, Criswell LA, Gregersen PK, Shadick NA, et al. The influence of polygenic risk scores on heritability of anti-CCP level in RA. Genes Immun. 2014;15:107-14.

21. Okada Y, Wu D, Trynka G, Raj T, Terao C, Ikari K, Kochi Y, Ohmura K, Suzuki A, Yoshida S, et al. Genetics of rheumatoid arthritis contributes to biology and drug discovery. Nature. 2014;506:376-81.

22. Irizarry RA, Ladd-Acosta C, Carvalho B, Wu H, Brandenburg SA, Jeddeloh JA, Wen B, Feinberg AP. Comprehensive high-throughput arrays for relative methylation (CHARM). Genome Res. 2008;18:780-90.

23. Magnusson PK, Almqvist C, Rahman I, Ganna A, Viktorin A, Walum H, Halldner L, Lundstrom S, Ullen F, Langstrom N, et al. The Swedish Twin Registry: establishment of a biobank and other recent developments. Twin Res Hum Genet. 2013;16:317-29.

24. Arnett FC, Edworthy SM, Bloch DA, McShane DJ, Fries JF, Cooper NS, Healey LA, Kaplan SR, Liang MH, Luthra HS, et al. The American Rheumatism Association 1987 revised criteria for the classification of rheumatoid arthritis. Arthritis Rheum. 1988;31:315-24.

25. Ladd-Acosta C, Aryee MJ, Ordway JM, Feinberg AP. Comprehensive high-throughput arrays for relative methylation (CHARM). Curr Protoc Hum Genet. 2010; Chapter 20:Unit 20.1.1-19.

26. Rakyan VK, Down TA, Balding DJ, Beck S. Epigenome-wide association studies for common human diseases. Nat Rev Genet. 2011;12:529-41.

27. Aryee MJ, Wu Z, Ladd-Acosta C, Herb B, Feinberg AP, Yegnasubramanian S, Irizarry RA. Accurate genome-scale percentage DNA methylation estimates from microarray data. Biostatistics. 2011;12:197-210.

28. Benjamini Y, Hochberg Y. Controlling false discovery rate: a practical and powerful approach to multiple testing. J R Stat Soc B. 1995;57:289-300.

29. McLean CY, Bristor D, Hiller M, Clarke SL, Schaar BT, Lowe CB, Wenger AM, Bejerano G. GREAT improves functional interpretation of cis-regulatory regions. Nat Biotechnol. 2010;28:495-501. 
30. Reinius LE, Acevedo N, Joerink M, Pershagen G, Dahlen SE, Greco D, Soderhall C, Scheynius A, Kere J. Differential DNA methylation in purified human blood cells: implications for cell lineage and studies on disease susceptibility. PLoS One. 2012;7:e41361.

31. Houseman EA, Accomando WP, Koestler DC, Christensen BC, Marsit CJ, Nelson HH, Wiencke JK, Kelsey KT. DNA methylation arrays as surrogate measures of cell mixture distribution. BMC Bioinf. 2012;13:86.

32. Efron B, Tibshirani RJ. An introduction to the bootstrap. London: Chapman \& Hall/CRC; 1991.

33. Edgington ES. An additive method for combining probability values from independent experiments. J Psychol. 1972;80:351-63.

34. Hedges LV, Olkin I. Statistical methods for meta-analysis. San Diego: Academic Press; 1985.

35. Bock C. Analysing and interpreting DNA methylation data. Nat Rev Genet. 2012:13:705-19.

36. Tsai PC, Bell JT. Power and sample size estimation for epigenome-wide association scans to detect differential DNA methylation. Int J Epidemiol. 2015;44(4):1429-41.

37. Jaffe $A E$, Murakami $P$, Lee $H$, Leek JT, Fallin MD, Feinberg AP, Irizarry RA. Bump hunting to identify differentially methylated regions in epigenetic epidemiology studies. Int J Epidemiol. 2012;41:200-9.

38. Pesarin F, Salmaso L. Permutation tests for complex data: theory, applications and software. West Sussex: Wiley; 2010.

39. Staals RH, Pruijn GJ. The human exosome and disease. Adv Exp Med Biol. 2011;702:132-42

40. Yagi T. Clustered protocadherin family. Dev Growth Differ. 2008;50 Suppl 1: S131-40.

41. Raychaudhuri S, Sandor C, Stahl EA, Freudenberg J, Lee HS, Jia X, Alfredsson L, Padyukov L, Klareskog L, Worthington J, et al. Five amino acids in three HLA proteins explain most of the association between MHC and seropositive rheumatoid arthritis. Nat Genet. 2012;44:291-6.

42. Karouzakis E, Gay RE, Michel BA, Gay S, Neidhart M. DNA hypomethylation in rheumatoid arthritis synovial fibroblasts. Arthritis Rheum. 2009;60:3613-22.

43. Moran S, Arribas C, Esteller M. Validation of a DNA methylation microarray for $850,000 \mathrm{CpG}$ sites of the human genome enriched in enhancer sequences. Epigenomics. 2016:8:389-99.

44. Lefebvre JL, Kostadinov D, Chen WW, Maniatis T, Sanes JR. Protocadherins mediate dendritic self-avoidance in the mammalian nervous system. Nature. 2012;488:517-21.

45. Vazquez-Cintron EJ, Monu NR, Burns JC, Blum R, Chen G, Lopez P, Ma J, Radoja S, Frey AB. Protocadherin-18 is a novel differentiation marker and an inhibitory signaling receptor for CD8+ effector memory $T$ cells PLoS One. 2012;7:e36101.

46. Blin J, Fitzgerald KA. Perspective: The RNA exosome, cytokine gene regulation and links to autoimmunity. Cytokine. 2015;74:175-80

47. Basu U, Meng FL, Keim C, Grinstein V, Pefanis E, Eccleston J, Zhang T, Myers $D$, Wasserman CR, Wesemann DR, et al. The RNA exosome targets the AID cytidine deaminase to both strands of transcribed duplex DNA substrates. Cell. 2011;144:353-63.

48. Brouwer R, Vree Egberts WT, Hengstman GJ, Raijmakers R, van Engelen BG, Seelig HP, Renz M, Mierau R, Genth E, Pruijn GJ, van Venrooij WJ. Autoantibodies directed to novel components of the PM/Scl complex, the human exosome. Arthritis Res. 2002;4:134-8.

49. Clark PM, Dawany N, Dampier W, Byers SW, Pestell RG, Tozeren A. Bioinformatics analysis reveals transcriptome and microRNA signatures and drug repositioning targets for IBD and other autoimmune diseases. Inflamm Bowel Dis. 2012;18:2315-33.

50. Edgar R, Domrachev M, Lash AE. Gene Expression Omnibus: NCBI gene expression and hybridization array data repository. Nucleic Acids Res. 2002;30:207-10.

\section{Submit your next manuscript to BioMed Central and we will help you at every step:}

- We accept pre-submission inquiries

- Our selector tool helps you to find the most relevant journal

- We provide round the clock customer support

- Convenient online submission

- Thorough peer review

- Inclusion in PubMed and all major indexing services

- Maximum visibility for your research

Submit your manuscript at www.biomedcentral.com/submit
Biomed Central 